\title{
LA TEMPORALIDAD COMO ESTRUCTURA DE L'ACTION (1893) DE MAURICE BLONDEL
}

\section{Patricio Perkins*}

\section{Resumen}

El trabajo analiza la estructura de L'Action (1893) del filósofo francés Maurice Blondel en tres niveles diferentes según el modelo de la configuración triádica de la temporalidad. El primer nivel está implícito en la analítica de la acción llevada a cabo en la Introduction a la obra; el segundo nivel se identifica con el análisis de la pasividad del primer momento de la cuarta parte; el tercer nivel abarca la obra considerada en su globalidad, extendiéndose a la problemática del fenómeno de la acción, de la demostración del único ser necesario y de la alternativa de la acción. El análisis pretende demostrar que las dimensiones antecedente, presente y adveniente de la temporalidad están a la base de la comprensión de esta obra blondeliana.

Palabras clave: Blondel, acción, temporalidad, estructura, ser, conocimiento

\section{Abstract}

This paper aims to offer an analysis of the estructure of Maurice Blondel's L'Action (1893) in three different levels taking into account as a model the triadic configuration of temporality. The first level is implicit in the analysis of action made in the Introduction to the work; the second level consists in the analysis of passivity described in the first moment of the fourth part; the third level contains the whole work in its global sense, extending itself to the phenomenon of action, the argument about the only necessary being and the alternative of action. I pretend to prove that the past, present and for-coming dimensions of temporality are at the base of the comprehension of this work.

Keywords: Blondel, action, temporality, structure, being, knowledge

* Licenciado en Filosofía por la Universidad del Norte "Santo Tomás de Aquino". Actualmente cursa su Doctorado en Filosofía en la Universidad Católica de Santa Fe. Es becario doctoral del CONICET con lugar de trabajo en la Academia Nacional de Ciencias de Buenos Aires y miembro del Círculo de Fenomenología y Hermenéutica de Santa Fe y Paraná. Dirección electrónica: patricio.perkins@gmail.com 
La estructura sistemática de L'Action (1893) puede ser abordada desde múltiples perspectivas ${ }^{1}$. Me interesaría analizarla a partir de la repetición variada de una estructura triádica inspirada en la forma temporal de la existencia humana. Pretendo mostrar, por un lado, que el rasgo formal de esta estructuración temporal se mantiene idéntico a lo largo de las repeticiones; por otro lado, que el rasgo material de esta estructura varía levemente según la ubicación en donde se encuentre. La estructura formal consiste en la unidad de las tres dimensiones de la temporalidad que llamo respectivamente "antecedente", "presente" y "adveniente". Utilizo el neologismo "adveniente" para indicar el movimiento de retorno imprevisto que todo acto humano acarrea. Correlativamente usaré los neologismos "antecedencia" y "adveniencia" para caracterizar las dimensiones laterales a la presencia. Los niveles donde Blondel realiza una explícita analítica de la acción según esta estructura triádica son tres: primero, la introducción a L'Action; segundo, el primer momento de la cuarta parte; tercero, el argumento total de la obra en su sentido global. Es posible mencionar un cuarto nivel implícito de esta analítica: la tríada de capítulos que conforman cada etapa de la tercer parte. Dejo de lado este cuarto nivel, porque por su extensión requiere un estudio aparte. Con este artículo pretendo dar un paso previo para la confrontación de la filosofía blondeliana con los estudios hermenéuticos acerca de la temporalidad, tal como ya fue sugerido hace tiempo por José Gaos².

1 Otras perspectivas: Peter Henrici, "Les structures de L'Action et la pensée française", en Dominique Folscheid (ed.), Maurice Blondel: Une dramatique de la modernité. Actes du colloque d'Aix-en-Provence, mars 1989, Paris, Éd. Universitaires, 1990, pp. 32-43; Peter Henrici, "Blondels „Action" im Lichte der klassischen deutschen Philosophie", Theologie und Philosophie, Vol. 64, 1989, pp. 161-178; Raymond Saint-Jean, La genèse de L'Action: Blondel 1882-1893, Paris, Desclée de Brouwer, 1965; Micheal Conway, "A positive phenomenology: The structure of Maurice Blondel's early philosophy", The Heythrop Journal, Vol. 47, 2006, pp. 579600.

2 Cfr. José Gaos, "Consideraciones del traductor" en Martin Heidegger, Ser y Tiempo, trad. José Gaos, Buenos Aires, FCE, 2004, p. 7. 


\section{La Introduction à L'Action (1893).}

El programa de L'Action consiste en inaugurar "une sorte de Métaphysique à la seconde puissance" 3 . "Oui ou non, la vie humaine a-t-elle un sens, et l'homme a-t-il une destinée? J'agis, mais sans même savoir ce qu'est l'action, sans avoir souhaité de vivre, sans connaitre au juste ni qui je suis ni même si je suis. (...) J'en aurai le cœur net. S'il y a quelque chose à voir, j'ai besoin de le voir"'4.

L'Action comienza con la instalación de un asunto monádico que pretende abarcar la amplitud última de la problemática humana como un horizonte ineludible e infranqueable. El análisis semántico de "destinée" muestra el suelo natural del conflicto sistemáticamente abordado por Blondel: por un lado, indica un poder soberano regulador de los acontecimientos: el hado o el fato; por el otro, el curso de la existencia, en cuanto forjada por las decisiones del agente 5 . A la pregunta destinal se enfrenta el contenido mínimo y único desde el cual se comprende la pregunta: la acción, el instrumento hermenéutico capaz de desplegar el contenido totalizante del problema, porque ella misma es totalizante. La pregunta destinal, siendo la pregunta a la que reenvían todas las preguntas y desde la cual toman fuerza y sentido todas ellas, es eminentemente arquitectónica y obliga a una respuesta en primera persona ${ }^{6}$. La elección de la acción como punto de partida y la perspectiva egoica le valieron a Blondel una interesante comparación con el cogito cartesiano ${ }^{7}$. Además, desde la pregunta destinal emerge la noción de necesidad como un besoin: una necesidad radical de la vida para consigo misma.

${ }^{3}$ Mauriçe Blondel, "L'Action (1893)", en Euvres complètes. Vol. I., 1893. Les deux thèses, texte établi et présenté par Claude Troisfontaines, Paris, P.U.F., 1995, p. 498 [464].

${ }^{4}$ Idem, p. 15 [VII].

${ }^{5}$ Cfr. Idem, p. 504 [470].

${ }^{6}$ Cfr. André Hayen, "La philosophie de Blondel au temps de la $1^{\text {cr }}$ «Action»", Revue de Philosophie de Louvain, Vol. 59, 1961, p. 267.

7 Cfr. Jean-Luc Marion, "La conversion de la volonté selon L'Action", en Dominique Folscheid (dir.), Maurice Blondel: Une dramatique de la modernité, ed. cit., p. 155. 
El primer nivel de análisis de la acción según la configuración temporal se encuentre en la Introducción a L'Action (1893), donde Blondel utiliza tres términos para explicar la experiencia nucleada de modo irrefutable en la acción: la tríada fait, necessité y obligation. La acción, en primer lugar, es "un fait, le plus général et le plus constant de tous, l'expression en moi du déterminisme universal", se produce "sans moi"8. El término "fail" proviene del participio pasado de faire, denotando así la dimensión antecedente de la acción. Esta primera dimensión se enlaza al principio de mis actos: yo no decidí existir; por tanto, es un hecho, algo dado. La acción, en segundo lugar, es "une nécessité, que nulle doctrine ne nie puisque cette négation exigerait un suprême effort", se da "malgré moi". Esta segunda caracterización se anuda al empleo de mis actos, ya que estoy obligado a actuar incluso si decidiese quitarme la vida. La acción no puede retroceder detrás de sí, porque todo retroceder en la acción es un avanzarla. Aquí se juega la dimensión presente de la acción. La acción, en tercer lugar, es "une obligation; il faut qu'elle se produise par moi, même alors qu'elle exige de moi un choix douloureux, un sacrifice, une mort", se realiza "par moi"10. El sacrificio de toda acción consiste en la inevitable clausura que acarrea elegir una posibilidad entre muchas. Cuando actúo clausuro una infinidad de vías para siempre y engendro una consecuencia que escapa de mi control. Esta es la dimensión que no se ata ni a lo antecedente, ni a lo presente, sino a lo advenidero siempre a la obra en el actuar. La acción sintetiza mi ser implicándome y, por ende, se da a través de mí; por tanto, la acción siempre promueve todo el yo transformándolo.

Resumiendo, la acción se da a una con el yo según estos tres horizontes internos de antecedencia, presencia y adveniencia, cuya estructura viene caracterizada materialmente por las nociones correlativas de fait-sans moi-, necessité - malgré moi-y obligation -par moi-. Esta es la primera analítica de la acción a partir de la temporalidad de la existencia.

\footnotetext{
${ }^{8}$ Maurice Blondel, "L'Action (1893)", op. cit., p. 15 [VII].

${ }^{9}$ Ibidem.

${ }^{10}$ Ibidem.
} 


\section{La estructura temporal en cuanto pasividad de la acción.}

El segundo nivel se encuentre en el primer momento de la cuarta parte, donde se abordan los rasgos de pasividad inherentes a la acción y contrarios al impulso humano de autonomía. La interpretación blondeliana de la pasividad interna a la voluntad reproduce la triple dimensionalidad de la acción descrita en la introducción sólo en su estructura formal, permitiendo hablar de una triple opresión antecedente, presente y adveniente; no obstante, el contenido asignado a cada dimensión es ligeramente diverso.

Aquí la dimensión antecedente se refiere a una pasividad activa. ¿Qué significa esta paradoja? Por un lado, si se destaca la pasividad activa, entonces se indica que la voluntad no quiso querer: “(...) il veut; mais il n'a pas voulu vouloir"11. La pasividad antecedente repercute en la dimensión presente de la acción, volviendo inútil la acumulación o expansión, pues en lo querido nunca logra remontar hasta su origen. De tal modo, la inquietud original del hombre echa raíces en la antecedencia que, por un contragolpe necesario, tiñe de insuficiencia la dimensión presente de la acción. Por otro lado, si se destaca la pasividad activa, entonces se indica que la voluntad no quiso querer. Esto significa que la pasividad implica la necesidad de actuar, la voluntad no puede permanecer en sí misma por propia elección. Estar forzado a actuar es el rasgo que determinaba la dimensión presente de la acción en el anterior nivel, mientras que en éste se ubica en la dimensión antecedente.

La pasividad en la dimensión presente tiene dos aristas: por un lado, la resonancia de la pasividad antecedente repercute en la acción presente, volviendo todo lo querido una aproximación, y toda aproximación un abismarse en una lejanía inalcanzable; por otro lado, en el presente acontece el sufrimiento irreductible a un simple hecho observable, una consecuencia razonable o un medio querido. En el sufrimiento hay una inexplicable oposición real a la voluntad. El dolor se manifiesta a través de tres modos de desproporción: cuando se sufre lo que no se quiere, cuando no se hace todo lo que se quiso y cuando se hace lo que no se quiso y se lo termina

11 Idem, p. 360 [326]. 
queriendo. El mal puede ser interno o externo: se presenta como exterior, cuando se sufre lo que no se quiso, o como interior, cuando en lo querido, se encuentra algo no querido ${ }^{12}$.

La pasividad en la dimensión adveniente consiste en la sujeción irremediable a los efectos de las acciones, ya sea en las obras exteriores, ya sea en las sedimentaciones interiores. La inserción de la acción en el universo no se da sin el advenimiento de una respuesta imprevisible y diferente al acto. Se puede decir, aunque Blondel no use estos términos, que el advenimiento acontece sólo a condición de que (i) el mismo hombre devenga y (ii) de que sus obras le sobrevengan. Por un lado, el advenir es un devenir en cuanto la acción vuelve como sedimentación obligada y, por el otro, es un sobrevenir en cuanto las obras de la acción escapan a su dominio y se vuelven algo independiente del autor.

La diferencia entre el contenido de este nivel con respecto al anterior reposa principalmente en la dimensión presente. Ella se vuelve aquí el lugar de la insuficiencia de la acción y de la presencia del mal, mientras que en el primer nivel es caracterizada por la necesidad de actuar. La dimensión antecedente varía en su contenido en cuanto se le asigna como rasgo la necesidad de actuar y se despliega el corolario de la insuficiencia. La dimensión adveniente se aclara aquí más extensamente a través del devenir y sobrevenir del advenimiento. Aquí la estructura temporal tomada en su unidad funciona como la afirmación completa y detallada de la original impotencia humana. La conciencia de la estructura agudiza la conciencia de la impotencia y conlleva el verdadero sentimiento de soledad ${ }^{13}$.

\section{La estructura triádica en la globalidad de $L^{\prime}$ Action (1893).}

El tercer nivel revela la estructura temporal triádica subyacente a la obra misma tomada en su globalidad. La sección más adecuada para comprender esto es la más compleja del libro: me refiero al tercer capítulo

12 “Subir ce qu'on ne veut pas, ne pas faire tout ce qu'on veut, faire ce qu'on ne veut pas et finir par le vouloir, jamais on n'échappe entièrement à cette fatalité humiliante et douloureuse" (Idem, p. 363 [329]).

${ }^{13}$ Idem, p. 366 [332]. 
de la quinta parte ${ }^{14}$. Este abordaje supone trazar la coordinación entre la estructura temporal triádica y los temas definitivos de L'Action: el significado de la existencia objetiva y el sentido del ser.

Se pueden distinguir cinco instancias fundamentales en el desarrollo de L'Action: (i) la intencionalidad de la acción afirmada como conclusión de la crítica al esteticismo; (ii) la dirección positiva de la voluntad, conclusión de la crítica al pesimismo; (iii) la insuficiencia de la autoconfiguración fenoménica de la intencionalidad, conclusión de la tercera parte (que podría concebirse como una crítica a un positivismo ampliado); (iv) la dirección supra-fenoménica de la intencionalidad, i.e. la afirmación del único Ser necesario; (v) la ineludible alternativa entre la vida y la muerte de la acción. La fenomenología de la acción, i.e. el despliegue impersonal de la acción por dentro de la epochè ontológica propuesta por Blondel en la introducción a su obra, se extiende a las cinco instancias, lo cual no debe confundirse con una homologación entre ellas ${ }^{15}$. Si aplicamos la estructura tríadica temporal a estas cinco instancias, el resultado es el siguiente.

La antecedencia atañe a los tres niveles que conforman "la zona neutra de la ciencia"16: (i) la intencionalidad de la acción, (ii) la dirección positiva de la intencionalidad, (iii) la autoconfiguración fenoménica de la dirección positiva de la intencionalidad. La cuarta instancia funda la dimensión presente y la quinta la dimensión adveniente. Cada instancia conlleva una determinación esencial de la acción y un orden de fundamentación escalonado.

La dimensión antecedente se identifica justamente con lo que Blondel llama el conocimiento subjetivo y propulsivo anterior a la opción ${ }^{17}$, que llamaré conocimiento subjetivo I (más adelante definiré el conocimiento subjetivo II ${ }^{18}$. Esta dimensión contiene la implicación espontánea de toda

14 "Ce chapitre extrêmement dense et difficile peut être considéré comme une relecture de l'oeuvre mise à l'intérieur de l'oeuvre" (Claude Troisfointaines, "Notice", en Maurice Blondel, "L'Action (1893)", op. cit., p. 10).

${ }^{15}$ Maurice Blondel, "L'Action (1893)", op. cit., pp. 486 [452], 492 [458].

${ }^{16}$ Idem, p. 520 [486].

${ }^{17}$ Cfr. Idem, p. 471 [455].

${ }^{18}$ Cfr. Idem, pp. 484-485 [450-451]. 
acción, definible como la aparición del objeto en cuanto independiente de nuestro poder, i.e. como una presencia necesaria en nuestra acción. Las cosas nos aparecen como un objeto producido espontáneamente por nuestro pensamiento bajo una serie inteligible de causas eficientes y, al mismo tiempo, como término de nuestro querer dentro de un sistema de causas finales. De este modo, todo objeto es concebido como independiente de nosotros porque debe ser libremente querido en el interior de una cadena de fenómenos solidarios y heterogéneos, cuyas oposiciones por su carácter fenoménico nunca son contradictorias. La idea necesaria de realidad objetiva, i.e. independiente de nosotros, trae aparejada como efecto la subordinación de esta realidad al uso de la voluntad. Este conocimiento se identifica con la afirmación del ser anterior a toda posible negación, con la representación del ser, con la idea del objeto, i.e. con la producción necesaria del conocimiento y la implicación inevitable del orden entero de los fenómenos. La dimensión antecedente desplegada en la totalidad de sus implicancias pone de manifiesto que el principio de la serie fenoménica está más allá del horizonte mundano, introduciendo así el contenido de la dimensión presente.

La dimensión presente atañe al "entre" que Blondel ubica en medio del conocimiento subjetivo I y II. Esta dimensión es el ámbito donde acontece la dirección al ser (recordemos que Blondel distingue entre dirección a los fenómenos y al ser) y, por ende, el gozne entre la fenomenología de la acción y la efectiva posesión o privación del ser posterior a la alternativa. Está entre lo previo a la opción (la representación del ser) y lo posterior a la opción (la posesión del ser), i.e. entre el planteo necesario del problema y la traducción de la solución en el sistema total de la existencia. Esta dimensión se identifica con lo que Blondel entiende como el espacio propiamente objetivo: el "entre" ubicado en el medio de la pasividad constrictiva de los fenómenos que se imponen a la voluntad y la iniciativa subjetiva que integra todo el orden de fines en el ser del hombre. La realidad del fenómeno no se halla ni en la inmanencia del objeto en la razón, ni en la inmanencia de la razón en el objeto, sino en el entre mediador de uno y otro. Esto significa que la presencia real es el punto de encuentro entre la implicación espontánea de toda la serie fenoménica y la necesidad de ratificarla por un libre sí o no. La dimensión presente es el ámbito de la 
acción en cuanto mediadora entre el conocimiento del ser y el ser en el conocimiento.

El contenido de la dimensión presente se inicia en el conflicto ("antibolie") entre el querer bastarse y no ser capaz de ello que impuso el análisis de la dimensión antecedente ${ }^{19}$. La antibolía es introducida como ligamen entre la dimensión presente y la antecedente, pues es conclusión de una e inicio de la otra. Para Blondel el aparente fracaso de la acción sólo es posible si implica un horizonte de anticipación afectivo a priori dentro del cual se da el contraste entre la volonté voulante y la volonté voulue. La estructura triádica de la pasividad - primer momento de Le conflit- recubre una condición positiva superior y englobante. La experiencia del sufrimiento sólo es posible por una conciencia que abarque como contra-polo la percepción del flujo de los fenómenos y la comprensión de los fenómenos en cuanto fenómenos, es decir, de aquello que no se basta a sí mismo ${ }^{20}$. Es necesario, entonces, volverse a lo otro diverso de los fenómenos, sin lo cual ellos no serían, y sin los cuales él no sería conocido, "cette chose qui semble néant puisqu'elle est hors des phénomènes" 21.

Entonces, dentro de la dimensión antecedente se determina la imposibilidad de detenerse -posición necesaria de la intencionalidad-, de retroceder -inevitable direccionalidad positiva- y de circunscribir el querer humano a la positividad fenoménica. La dimensión presente se hace cargo de esta triple imposibilidad a través de la formulación consciente de la antibolía: desde este conflicto emerge como un pasaje obligado en la conciencia el reconocimiento del único ser necesario: "Oui il faut que je me veuille moi-même; or, il m'est impossible de m'atteindre directement; de moi à moi, il y a un abîme que rien n'a pu combler" 22 . El paso hacia las demostraciones del único ser necesario es, entonces, un retroceso para alcanzar lo que ya estaba al inicio.

\footnotetext{
${ }^{19}$ Idem, p. 357 [323].

${ }^{20}$ Idem, p. 368 [334].

${ }^{21}$ Idem, p. 370 [336].

22 Idem, p. 372 [338].
} 
Blondel sólo estudia el asunto del único ser necesario en la medida en que es una presencia en nuestra conciencia actuante ${ }^{23}$. La afirmación del único ser necesario se encuentra en un cierto medio, ápice del desarrollo de Le phénomène de l'action y causa de un desarrollo ulterior en L'Alternative. Blondel había intitulado esta parte "Partie décisive" en lugar de "L'être nécessaire de l'action", cambiando los títulos justo antes de que se imprimieran ${ }^{24}$. La decisividad de esta parte descansa en que transforma la pura descripción fenomenológica de la acción en una verdadera dialéctica que define el ser del hombre ${ }^{25}$. La dimensión presente, entonces, se refiere a la intersección o pasaje entre fenomenología, como conocimiento del ser y ontología como posesión del ser. Atañe, entonces, al conflicto que se abre por la dirección supra-fenoménica de la voluntad. Es por ello que se encuentra en Blondel una vacilación a la hora de ubicar las pruebas de Dios en el desarrollo global de su argumento. La razón descansa en que yacen en un cierto medio entre la expresión fenoménica de la acción y su fijación ontológica definitiva. En efecto, Blondel, por un lado, no las menciona al describir la zona neutra de la ciencia ${ }^{26}$; pero, por el otro, las describe como un anillo más en la descripción fenoménica de la serie de medios que la acción se auto-impone ${ }^{27}$. Esta vacilación responde a la idea que tiene Blondel de objetividad y verdad: para Blondel la verdad real de los objetos consiste en lo que depende de nosotros querer o no dentro de las

${ }^{23} \mathrm{El}$ análisis de las pruebas de Dios, del mismo modo que el resto de los análisis temáticos, se dirige en este artículo sólo a la manifestación de la estructura subyacente. No pretendo probar ni la legitimidad ni la perfección de la argumentación blondeliana en torno a este tema.

24 Raymond Saint-Jean, op. cit., p. 208.

${ }^{25}$ Idem, p. 232.

26 Cfr. Idem, p. 517-520 [483-486]. En estas páginas describe de modo pormenorizado la zona neutra de la ciencia bajo la épochè ontológica.

27 "C'est une nécessité d'abord que l'ordre entier des choses, tel que la science de l'action l'a peu à peu déployé devant la connaissance réfléchie, ait, dans sa totalité, la même valeur objective: un anneau ne saurait y ĉtre moins solide, moins nécessaire, moins réel qu'un autre. Et malgré l'extrême diversité des éléments qui composent la série, (...) tous, aussi bien l'affirmation du Dieu vivant que le phénomène physique le plus brut, ne sont encore que des formes d'un même besoin intérieur (...)." (Maurice Blondel, "L'Action (1893)", op. cit., p. 492 [458]). 
condiciones necesarias. Esto no es otra cosa que la realidad de donde derivan las necesidades mismas afirmada en las pruebas de Dios. Como todo depende de la postura tomada delante del único ser necesario, principio de toda la serie de seres que la acción nos impone, éste se considera dentro de la serie impuesta por la acción, aunque sólo a condición de ocupar el lugar de principio de la serie al que conduce todo el determinismo de medios y que produce como efecto la bifurcación en la alternativa entre la vida o la muerte de la acción.

Del mismo modo que la cumbre de la dimensión antecedente afecta la esencia de la dimensión presente, la implicación última de ésta se articula con la dimensión adveniente. Las pruebas de Dios son el horizonte presente de la acción que, habiendo retomado toda la serie fenoménica en su antecedencia hasta la posición ineludible del conflicto, reviste una significación a partir del instante. Las vías no son una mediación más, homóloga a la serie fenoménica de la tercera parte, sino la dimensión presente ineludible de la acción, i.e. el contenido real del instante.

Describiré brevemente las pruebas. Blondel aborda cuatro pruebas diversas enlazadas sucesivamente: primero, la llamada "la voie du néant" 28; segundo, la cosmológica; tercero, el argumento teleológico; y, cuarto, el ontológico. La vía de la nada es una suerte de contraprueba ontológica, porque funda la imposibilidad del no-ser absoluto a partir de la insuficiencia del ser relativo. La nada se ofrece originalmente a la conciencia como la negación de "tout ce qui est objet immédiat de connaissance et de désir"29. Si abismarse sobre la nada y afirmarla es implícitamente operar una negación sobre la totalidad de lo percibido y querido, entonces tal negación hace resaltar por contrapeso la grandeza del negador, trascendente a la totalidad del orden fenoménico. De tal modo, pensar la nada es, como dice Blondel, "un hommage à l'être" 30 , esto es, reconocer que lo esencial de la aspiración del hombre supera lo percibido. De allí que lo negativo resulte adecuado para penetrar en el misterio presente en nuestros actos.

\footnotetext{
${ }^{28}$ Idem, p. 375 [341].

${ }^{29}$ Ibidem.

30 Idem, p. 376 [342].
} 
Sin embargo, esta peculiar contra-vía ontológica fundada en lo que parece no ser exige un complemento que le dé sentido y precisión. El complemento es la prueba cosmológica, que afirma el ser a partir de la totalidad de lo que parece ser, ya que la plenitud de la nada y el ser que esconde sólo se manifiestan "par l'usage des phénomènes et l'épreuve de leur insuffisance" 31 . La voluntad toma conciencia de su porte, es decir, opera una reconducción hacia ella misma y hacia sus exigencias inapelables a través del orden entero de los fenómenos, al que no puede renunciar, como tampoco atenerse: he aquí el pasaje a la prueba cosmológica. Es imposible renunciar a la mediación fenoménica, como también lo es detenerse sólo en ella. La experiencia común acusa esta prueba a través de la ambigüedad de los actos que manifiestan simultáneamente "une étrange indigence" y "une plus étonnante plénitude" 32: esta ambigüedad desconcertante de lo contingente exige el apoyo en lo absolutamente necesario. Toda la fuerza del argumento cosmológico reposa en que lo querido supone que el único necesario es a partir de que queremos los fenómenos y aún más.

Ahora bien, ¿qué es esta " $x$ " necesaria? Hasta aquí, no es ni los fenómenos ni la nada, aunque sea sólo concebible a través de ellos. Evidentemente se debe partir desde su presencia en el yo, porque es imposible partir desde la " $\mathrm{x}$ ". Blondel elige el continuo circuito entre pensamiento y acción en el hombre como experiencia que indica la intimidad del único ser necesario. El circuito entre el pensar y el actuar atraviesa siempre un claro que no se da por nuestra acción o pensamiento. En efecto, incesantemente concebimos un ideal que, al ejecutarlo, lo superamos y, a su vez, en la misma ejecución irrumpe una nueva proyección. La constatación de la coincidencia oscilante entre acción y pensamiento indica una eficacia que no tiene su principio en la acción ni en el pensamiento del hombre. La sabiduría y el poder se dan en el hombre a través de un pasaje incesante; de tal modo, se descubre la " $\mathrm{x}$ " misteriosa como una realidad potente y sabia que sobrepasa infinitamente al hombre.

${ }^{31}$ Idem, p. 377 [343].

32 Ibidem. 
No se debe concebir este pasaje simplemente como una coincidencia que a veces sucede y otras veces no, como afirma Dhotel ${ }^{33}$. La coincidencia inestable es el instante mismo, porque el pasaje oscilante es la esencia de la dimensión presente de la acción. De hecho, Blondel habla de "une disproportion constante" 34 . Blondel destaca dos corolarios a partir de esta prueba: primero, está en el hombre como una perfección inaccesible, como el "centre de ce que je pense et de ce que je fais" 35 ; segundo, el hombre no se confunde con esta presencia, ni ésta con aquel, porque está en él, pero no es de él, justamente porque no la puede igualar. La " $x$ " se reconoce como lo que no es capaz de hacer el hombre en lo que efectivamente hace: la identidad de lo ideal y lo real. Así, el único ser necesario permanece necesario e inaccesible, porque es aquello que yo no puedo hacer ni comprender, pero sin el cual yo no puedo ni actuar, ni pensar.

Siguiendo este orden en las pruebas, el argumento ontológico cobra su verdadero sentido, ya que ingresa en el encadenamiento de las pruebas como el reconocimiento de una idea de perfección viva en la conciencia: es un acto que permite actuar ${ }^{36}$. Esta idea representa la identidad entre pensamiento y acción que manifiesta la prueba teleológica como una certeza positiva en el hombre, aunque no del hombre. El término hacia el cual lanza este argumento es lo máximo y lo más íntimo: tal exceso lo hace inaccesible.

En este sentido, encuentro equivocada la tesis de Sadler ${ }^{37}$, según la cual hay una opción por el egoísmo o por la caridad previa al argumento

33 Cfr. J.-C. Dhotel, “Action et dialectique. Les Preuves de Dieu dans L'Action de 1893", Archives de Philosopbie, Vol. 26, 1963, p. 17.

${ }^{34}$ Maurice Blondel, "L'Action (1893)", op. cit., p. 378 [344]. Cfr. Idem, p. 380 [346]. 35 Ibidem.

${ }^{36}$ Una anotación de sus diarios complementa el sentido vital de esta prueba y su unidad con cl determinismo de la acción: "Dieu est présent dans l'idée que nous avons de lui. C'est tout le sens de la preuve ontologique. Il est lui-même idée: et l'idéal n'est que la poursuite inassouvie de cette idée, comme l'indéfini n'est que l'essai toujours infructueux de l'infini" (Maurice Blondel, Carnets Intimes (1883-1894), Éditions du Cerf, Paris, 1961, p. 161).

37 Gregory B. Sadler, "The Ontological Proof, the Option, and the Unique Necessaire: Maurice Blondel's Examination of the Proof in Anselm, Descartes, and Malebranche", The Saint Anselm Journal, Vol. 2, 2005, pp. 90-91. 
ontológico y que modifica concomitantemente la aproximación a aquel. Por el contrario, las pruebas abren la dimensión adveniente de la alternativa en pos de una autosuficiencia contradictoria o de la espera de una solución que no podrá brotar exclusivamente de energías humanas. La opción, en L'Action, es una opción delante de algo trascendente al horizonte mundano y las pruebas son justamente el esclarecimiento científico de la acción como relación con algo trascendente a la posibilidad de producción humana: el único ser necesario es la presencia de la alteridad irreductible a la acción del hombre. La alternativa sólo se abre luego de haber establecido metódicamente la dirección supra-fenoménica de la acción. No puede haber opción delante de lo trascendente antes de la demostración de su inevitable presencia en el mismo actuar -y la prueba ontológica forma justamente parte de esta demostración-; de otro modo, Blondel estaría cayendo en la crítica de petición de principio que le dirigió Boutroux en la defensa de tesis y que él decididamente refutó ${ }^{38}$. El gran problema se abre: Dios es aquello que no se puede ser ni hacer; y sin embargo, se tiene el ser y la acción sólo para aspirar a él. El contenido de la dimensión adveniente, consecuentemente, coincide con la alternativa. La opción es doble: "Vouloir et ne pas pouvoir, pouvoir et ne pas vouloir, c'est la option même qui s'offre à la liberté: 's'aimer jusqu'au mépris de Dieu, aimer Dieu jusqu'au mépris de soi"'39.

Esta alternativa es de suma importancia, porque es para Blondel el principio mismo que opera la síntesis de toda la vida humana, es decir, el sentido, el ejercicio pleno de la voluntad. Sin la explicitación de lo que envuelve necesariamente la opción de la voluntad, quedarían trunca la dimensión antecedente y presente de la acción. Blondel explica así el ligamen entre las tres dimensiones:

Le phénomène le plus rudimentaire ne serait pas ce qu'il est pour nous, sans le lien qui l'y rattache. En sorte que, réciproquement, la connaissance du moindre phénomène a pour rôle de nous faire remonter, par la chaîne

${ }^{38}$ Cfr. Maurice Blondel, "Une soutenance (manuscrit 1893)", op. cit., pp. 700-702.

39) Maurice Blondel, “L’Action (1893)”, op. cit., p. 355 [389]. 
du déterminisme, à cette libre décision d'où dépend la destinée de chacun ${ }^{40}$.

Toda la vida conduce entonces a una alternativa necesaria, en cuanto ya se halla envuelta en el conocimiento y en la afirmación del menor de los fenómenos, y, al mismo tiempo, alternativa libre, ya que la acción de la voluntad sancionará tal eficacia insuprimible.

La dimensión adveniente coincide con el conocimiento subjetivo II o lo que Blondel llama "vraiment une connaissance objective" 41 . Esta dimensión atañe al ser en el conocimiento posterior a la opción; por ende, a lo que depende del querer del hombre con respecto a la Alternativa. Aquí se halla el ámbito de lo objetivo en cuanto traducción en la vida entera de la solución tomada. En la adveniencia las cosas se dan como dependientes del empleo de la vida y, consecuentemente, el espacio de la libertad definida delante del único ser necesario. Esta dimensión se enlaza a la estructura triádica global a través de lo que Blondel llama "la acción necesaria" de la idea de Dios. ¿Por qué la idea de Dios tiene una acción necesaria? Porque es un momento en el dinamismo de la acción: salida de la acción, vuelve a la acción para transformarla. Siendo el pensamiento de Dios algo en la acción, tal trascendencia del pensamiento conduce a la trascendencia de la acción, ya que "ce que nous connaissons de Dieu est ce surcroît de vie intérieure qui réclame son emploi: nous ne pouvons donc connaître Dieu sans vouloir le devenir en quelque façon" 42 . En efecto, el sentido de las demostraciones de Dios es manifestativo, porque no probamos a Dios como una realidad extrínseca a la acción humana, sino como un pasaje inevitable del instante. En definitiva, ninguno de nosotros prueba a Dios, sino para probar a Dios. De alli que las pruebas sean el gozne hacia la alternativa decisiva entre la muerte en vida o la apertura última a un acto que no me puedo dar yo. Estas dos figuras que la voluntad extática reviste son, en definitiva, la de la voluntad de poder y la de una voluntad de impotencia. Así, la alternativa se

\footnotetext{
40 Ibidem.

${ }^{41}$ Idem, p. 472 [438].

42 Idem, p. 379 [413].
} 
opera necesariamente porque el único ser necesario es desde siempre una realidad operante en nuestro yo.

El conocimiento subjetivo II reviste, entonces, dos figuras: i) conocimiento privativo, ii) conocimiento constitutivo del Ser. El conocimiento privativo del ser consiste en aceptar los medios, pero no el fin, i.e. afirmar los seres, pero no el Ser. La descripción de la naturaleza de esta figura destaca desde la perspectiva negativa de la privación la situación peculiar del único ser necesario en el argumento blondeliano, ya que la diferencia específica entre ambas figuras descansa en la postura delante de éste. Según Blondel, en el conocimiento privativo, la voluntad continúa produciendo la exigencia de todo lo que debe ser el hombre para ser sí mismo, al mismo tiempo que el conocimiento muestra todo lo que excluye de necesario en la voluntad. No debe confundirse el conocimiento privativo con el conocimiento subjetivo I. El ser en el conocimiento privativo incluye de modo completo y positivo el conocimiento subjetivo I y forma el conocimiento subjetivo II como un todo negativo. El conocimiento constitutivo del Ser, en cambio, se identifica con la aceptación de las cosas y de su principio. Pero el modo de aceptar al único ser necesario pasa a través del anonadamiento y el sacrificio.

Como Dios se ofrece a la conciencia del hombre a través de la materia, por ende, es sólo a través de ella que puede el hombre ofrecerse a Dios: este medio entre Dios y el hombre es para Blondel la verdad real del fenómeno. La verdad absoluta de los objetos es justamente su rol mediador entre el hombre y el principio último de la realidad. La realidad objetiva se define, entonces, como la diferencia entre el conocimiento subjetivo I y II, como el entre en medio del padecimiento necesario del orden fenoménico implicado en la acción y la iniciativa propia que integra todo el sistema de fines en la propia vida.

\section{Conclusión.}

La estructura formal de antecedencia, presencia y adveniencia se reproduce en los tres niveles señalados con variaciones de contenido. El primer nivel de la "Introducción" identifica la antecedencia con la noelección de la existencia propia, la presencia con la necesidad de ejercer la 
acción y la adveniencia con la transformación propia y ajena que acarrea el actuar. El segundo nivel, centrándose en la experiencia de la pasividad, asigna a la antecedencia lo que se describió en el primer nivel como contenido de la dimensión antecedente y presente, a la presencia el lugar de la insatisfacción insuperable y del escándalo del sufrimiento, a la adveniencia el ámbito del devenir y sobrevenir de los actos. El tercer nivel, tomando una altura superior a los dos anteriores, despliega la presencia como el significado de la objetividad en cuanto intersección entre la antecedencia del conocimiento subjetivo I y la adveniencia del conocimiento subjetivo II. Sin lugar a dudas, este tercer nivel es el más importante al establecer la interpretación de las tres dimensiones de la acción en relación con el destino del hombre.

En los tres casos, Blondel intenta definir la triple-dimensionalidad del instante a través del cual la acción pasa incansablemente. Pero las dimensiones no valen ni son eficaces sino por su unidad sintética, que reproduce el movimiento de la vida ${ }^{43}$. Luz abismal a nuestras espaldas, acción en el instante urgente y eficacia inminente delante de la totalidad de lo real; de allí que haya en la acción un destino en juego, porque nadie quiere el mínimo sin remontarse hasta lo máximo. Cada fenómeno, según un orden de mediación graduado, cumple la función de signo inevitable de algo otro, al mismo tiempo que revelación del querer infinito en el hombre. Unidad, sentido y eficacia, la estructura triádica de L'Action inspirada en la forma de la temporalidad fundamenta el instante en su vinculación con el sentido total de la vida. Instante urgente, pues "[I]l tempo è caro/ in questo regno" 44 .

Recibido: 05/2010; aceptado: 06/2010.

43 "[L]a vérité nc vaut jamais que par l'unité totale de son expression" (Idem, p. 272 [238]).

${ }^{44}$ Alighieri, Dante, Divina Comedia, edición bilingüe a cargo de Ángel J. Battistessa, Asociación Dante Alighieri, Buenos Aires, 2003, p. 316 [XXIV, 91-92]. 